\title{
Long-Term Gynecological Outcomes in Women with Congenital Adrenal Hyperplasia due to 21-Hydroxylase Deficiency
}

\author{
T. H. Johannsen, ${ }^{1}$ C. P. L. Ripa, ${ }^{2}$ E. Carlsen, ${ }^{1}$ J. Starup, ${ }^{3}$ O. H. Nielsen, ${ }^{4}$ M. Schwartz, ${ }^{5}$ \\ K. T. Drzewiecki, ${ }^{6}$ E. L. Mortensen, ${ }^{2}$ and K. M. Main ${ }^{1}$ \\ ${ }^{1}$ University Department of Growth and Reproduction, GR-5064, Rigshospitalet, Blegdamsvej 9, DK-2100 Copenhagen, Denmark \\ ${ }^{2}$ Department of Health Psychology, Institute of Public Health, University of Copenhagen, DK-1014 Copenhagen, Denmark \\ ${ }^{3}$ University Department of Gynecology and Obstetrics, Rigshospitalet, DK-2100 Copenhagen, Denmark \\ ${ }^{4}$ University Department of Paediatric Surgery, Rigshospitalet, DK-2100 Copenhagen, Denmark \\ ${ }^{5}$ University Department of Clinical Genetics, Rigshospitalet, DK-2100 Copenhagen, Denmark \\ ${ }^{6}$ University Department of Plastic Surgery and Burn Unit, Rigshospitalet, DK-2100 Copenhagen, Denmark
}

Correspondence should be addressed to K. M. Main, katharina.main@rh.regionh.dk

Received 23 February 2010; Revised 26 April 2010; Accepted 22 August 2010

Academic Editor: Peter Allen Lee

Copyright ( $) 2010$ T. H. Johannsen et al. This is an open access article distributed under the Creative Commons Attribution License, which permits unrestricted use, distribution, and reproduction in any medium, provided the original work is properly cited.

\begin{abstract}
Background. Our knowledge on long-term outcome in CAH remains incomplete. Methods. In a prospective study (33 CAH patients, 33 age-matched controls), reproductive outcomes, self-rating of genital appearance and function, and sexuality were correlated to degree of initial virilisation, genotype, and surgery. Results. Patients had larger median clitoral lengths (10.0 mm [range 2-30] versus 3.5 [2-8], $P<.001)$, shorter vaginal length $(121 \mathrm{~mm}[100-155]$ versus 128 [112-153], $P=.12)$, lower uterine volumes (29.1 $\mathrm{ml}$ [7.5-56.7] versus 47.4 [15.9-177.5], $P=.009)$, and higher ovarian volumes (4.4 ml [1.3-10.8] versus 2.8 [0.6-10.8], $P=.09$ ) than controls. Satisfaction with genital appearance was lower and negatively correlated to degree of initial virilisation $\left(r_{s}=\leq-0.39, P \leq .05\right)$. More patients had never had intercourse $(P=.001)$, and age at 1st intercourse was higher (18 yrs versus 16 yrs, $P=.02$ ). Conclusion. Despite overall acceptable cosmetic results, reproductive outcomes were suboptimal, supporting that multidisciplinary teams should be involved in adult follow up of CAH patients.
\end{abstract}

\section{Introduction}

Congenital adrenal hyperplasia due to 21-hydroxylase deficiency is an autosomal recessive disorder characterized by impaired or total loss of activity in the enzyme required for cortisol and aldosterone production, resulting in highly increased adrenal androgen synthesis [1]. Depending on the severity of the underlying defect in CYP21, female $\mathrm{CAH}$ patients show varying degrees of genital virilisation. Ambiguous genitalia in the newborn, severe clitoromegaly, and vaginal stenosis may require many diagnostic and surgical procedures, not only initially, but also repetitively during adolescence. Despite an increasing focus on optimal treatment of CAH patients, our knowledge of long-term outcomes of this condition remains incomplete. Thus, the aim of the present study was to evaluate gynecological and reproductive outcomes in a group of women diagnosed with CAH due to 21-hydroxylase deficiency in childhood or adolescence.

\section{Materials and Methods}

\subsection{Population Characteristics}

2.1.1. Patients. This study was part of a follow-up study of adult female patients diagnosed with disorders of sex development (DSD) at the University Hospital of Copenhagen (Rigshospitalet) from 1953-2003. Participation rate was $81 \%$. In total, $33 \mathrm{CAH}$ patients with genetically verified CYP21 deficiency were included (30 yrs, 19-52). As previously reported $[2,3]$, patients with CYP21 deficiency were divided according to the clinical data and the predicted 
severity of the mutation [4] into salt-wasters (SW, $n=21$ ), simple-virilizers (SV, $n=6$ ), and late-onset (LO, $n=5) \mathrm{CAH}$. One patient was first diagnosed in adolescence and was in the present study included in the group of patients with lateonset CAH.

Diagnosis of CAH patients occurred at a median age of 0 yrs (0-16 yrs). At birth, seven patients with salt-wasting $\mathrm{CAH}$ were assigned male gender, but with a reassignment to females during the first months of life, while ten saltwasters and two simple-virilizers with ambiguous genitalia at birth were assigned female gender. Data on virilisation at birth was missing in two patients. In the patients, the degree of virilisation at the time of diagnosis was graded from 1 (lowest) to 5 (highest): (1) none; (2) virilisation diagnosed $\geq 1$ year of age; (3) virilisation diagnosed $<1$ year of age; (4) ambiguous genitalia at birth; (5) severe virilisation with male gender assignment at birth, but reassignment to female after diagnosis. In five patients, significant urogenital comorbidity included unilateral ureteronephrectomia due to a malformed kidney and hydronephrosis, hysterectomy, unilateral ovariectomy due to cysts, unilateral salpingooophorectomy due to pyosalpinx, and salpingectomy after an extrauterine pregnancy. Additionally three patients reported urine incontinence.

2.1.2. Controls. A control group was recruited through the Danish Civil Registration System. For each patient, twenty women living in the Copenhagen area and born in the same month and year as the patient were contacted by a letter. The control with the closest match for self-reported education was selected for pair-wise matching. Participation rate was $37 \%$. One control person had a duplication of the ureter and two had cervical conisations performed.

2.2. Assessment Program. Data were collected via medical records and a structured interview. Satisfaction with genital appearance and function was graded on an analogous 1-7 scale, with 1 representing not satisfied, 4 partly satisfied, and 7 satisfied. If participants were unable to answer, answers were treated as missing data.

The gynecological examination included measurements of clitoral length $(\mathrm{mm})$, and maximal vaginal length and diameter measured with Hegar instruments ( $\mathrm{mm}$, maximal vaginal width assigned $\geq 30 \mathrm{~mm}$ ). Uterine and ovarian size was determined by transvaginal ultrasonography with volumes calculated as an ellipsoid [5]: volume $(\mathrm{ml})=$ $4 / 3 * \Pi *$ length $(\mathrm{cm}) *$ width $(\mathrm{cm})^{2} * 1 / 8$. The mean value of the two ovaries was presented $(n=31)$, unless only one was visualized at ultrasound $(n=8)$. Six patients declined gynecological examination, and four patients had no gynecological examination performed due to logistical reasons. In two controls, measurements were excluded due to ongoing pregnancy.

The degree of hirsutism was graded according to the modified Ferriman-Gallwey score $[6,7]$, in which nine body areas were graded from minimal (grade 1) to frank hirsutism (grade 4), 0 indicated absence of hirsutism. Five patients declined to participate in this examination. Body mass index (BMI) was calculated as weight (in kilograms) divided by height (in meters) squared. Height was determined by a Harpenden stadiometer (Holtain Ltd, Crymych, UK) to the nearest $0.1 \mathrm{~cm}$, and weight was measured on a digital weight scale with a precision of $0.1 \mathrm{~kg}$ (SECA delta, model 707, SECA, Bradford, MA, USA). BMI measurements were excluded in the pregnant controls.

Hormone analysis parameters, which were not correlated to cycle day, included androgen status and antimüllerian hormone (AMH). The androgen status included serum testosterone, serum androstendione, and serum dihydrotestosterone (DHT), which were analyzed as described by Lykkesfeldt et al. [8], and serum dehydroepiandrosterone sulphate (DHEAS), which was measured by a radioimmunoassay. For testosterone, androstenedione and DHT, the intra- and interassay coefficients of variation were $5 \%$ and $<10 \%$, respectively, with a limit of quantification of $0.1 \mathrm{nmol} / \mathrm{L}$, while intra- and interassay coefficients of variation of DHEAS were $7 \%$ and $10 \%$, respectively, and the limit of quantification was $100 \mathrm{nmol} / \mathrm{L}$. The concentration of serum AMH was measured by an ultrasensitive sandwich enzyme immunometric assay using the EIA AMH/MIS kit (Immunotech A11893, Beckman Coulter, Marseille, France). For $\mathrm{AMH}$, the intra- and interassay coefficients of variation were $7.8 \%$ and $11.6 \%$, respectively, and the limit of detection was $2 \mathrm{pmol} / \mathrm{L}$.

2.3. Statistical Analyses. Nonparametric statistical analysis was used. The Mann-Whitney test with the Monte Carlo exact test was used in comparisons of patients and unmatched controls, the Wilcoxon signed rank sum test was used in comparisons of patients and matched controls, and the Kruskal-Wallis test in analysis of CAH subgroups. Correlations were done by Spearman correlation. Data are presented as medians (ranges), unless otherwise stated.

2.4. Approvals. Approvals were granted from the local ethical committee (01-051/01, 11-075/03), and the Danish Data Registry (2001-41-0689). The study was performed according to the Helsinki declaration II after written consent from the participants.

\section{Results}

Clitoral operations had been performed in $27 \mathrm{CAH}$ females, including reduction with preservation of the glans (SW: $n$ = 16, SV: $n=5$, LO: $n=1$ ) and amputation (SW: $n=4$, SV: $n=1)$. Five SW-patients and 1 SV-patient had clitoral operations performed twice. Age at first clitoral operation was 4.4 years (range $0.8-25$ yrs). Clitoral length was larger in $\mathrm{CAH}$ patients $(10 \mathrm{~mm}[2-30 \mathrm{~mm}])$ than in controls $(3.5 \mathrm{~mm}[2-8 \mathrm{~mm}]), P<.001$; see Figure 1. In patients, no associations were observed between clitoral length or clitoral surgery procedures and androgen levels, respectively, (data not shown). Serum-antimüllerian hormone (AMH) correlated inversely with clitoral length $\left(r_{s}=-0.59, P=\right.$ .01 ). No significant correlation to surgery or number of surgical procedures at clitoris was found $(P=.22$ and .13 , resp.), but age at clitoral surgery was inversely related to serum-AMH levels $\left(r_{s}=-0.57, P=.01\right)$. 


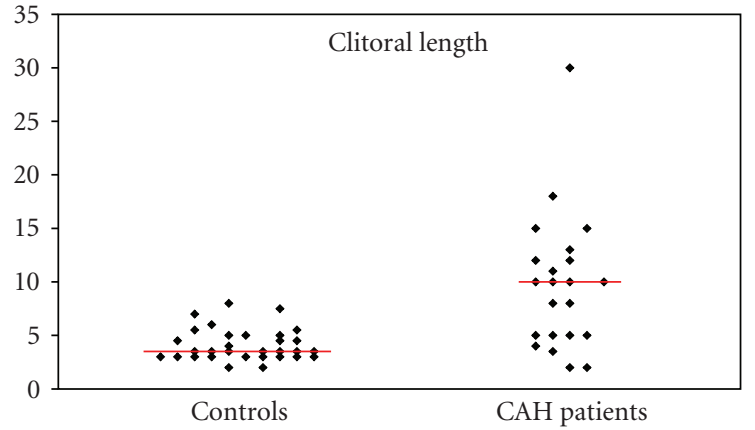

(a)

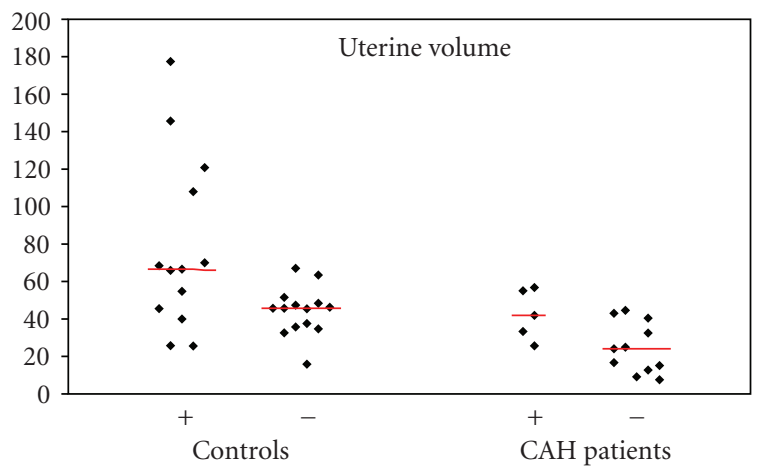

(c)

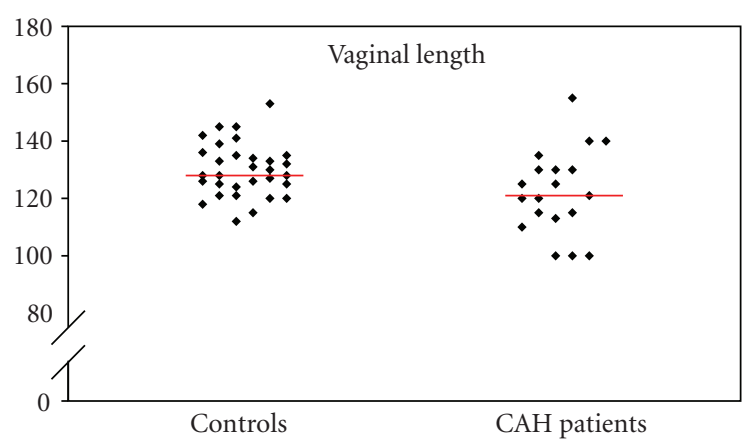

(b)

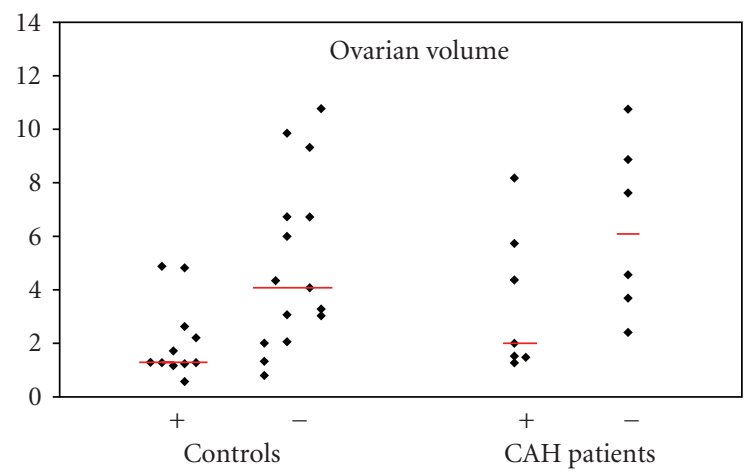

(d)

FIGURE 1: Gynecological outcomes in patients with congenital adrenal hyperplasia (CAH) and in controls: clitoral length (mm), vaginal length $(\mathrm{mm})$, uterine volume $(\mathrm{ml})$ separated by primipara/multipara $(+)$ or nullipara $(-)$, and ovarian volume $(\mathrm{ml})$ separated by current $(+)$ or no (-) use of hormonal contraception. Lines indicate median values.

TABLE 1: Satisfaction with appearance and function of external genitalia (mean and ranges, 1: lowest to 7: highest) in patients with congenital adrenal hyperplasia and in controls. Significance levels for differences between patients and controls are indicated as ${ }^{*} P<.05$ and ${ }^{* *} P<.01$.

\begin{tabular}{lcccr}
\hline & Controls & \multicolumn{2}{c}{ CAH patients } \\
& & All & Clitoral reduction & Clitoral resection \\
\hline Clitoral appearance & $6.8(5-7)$ & $5.8(1-7)^{* *}$ & $6.0(4-7)$ & $4.8(1-7)$ \\
Clitoral function & $6.9(6-7)$ & $6.2(1-7)^{*}$ & $6.2(2-7)$ & $5.4(1-7)$ \\
Vaginal appearance & $6.6(4-7)$ & $5.9(1-7)$ & $6.1(1-7)$ & $4.8(1-7)$ \\
Vaginal function & $6.5(3-7)$ & $6.0(1-7)$ & $6.4(1-7)$ & $5.0(1-7)$ \\
\hline
\end{tabular}

Vaginal operations had been performed in $24 \mathrm{CAH}$ patients, and patients were operated once (SW: $n=4, \mathrm{SV}$ : $n=2$, LO: $n=1$ ), twice (SW: $n=9$, SV: $n=2$ ), three times (SW: $n=4$ ), four times (SW: $n=1$ ), or five times (SW: $n$ $=1$ ), respectively. Vaginal operations included vaginoplasty with skin transplants from the thigh, buttock, perineum or labiae (SW: $n=18$, SV: $n=2$ ) or without skin transplants (SV: $n=1$, LO: $n=1$ ). Adequate data on vaginoplasty was missing in two patients ( $\mathrm{SW}=1, \mathrm{SV}=1)$. Age at first vaginal operation was 14.1 years (range 1.0-27.3). Vaginal length was nonsignificantly shorter in patients (121 mm [100-155 mm] than in controls (128 mm [112-153]), $P=.12$; see Figure 1. Thirty-six percent of the patients had a vaginal diameter below $30 \mathrm{~mm}$, as compared to $6 \%$ in the control group. No significant correlations were found in vaginal measurements and/or vaginal surgical procedures versus AMH-levels and androgen levels, respectively (data not shown).
In contrast to controls, significantly lower rating on genital outcome was reported in $\mathrm{CAH}$ patients with respect to clitoral appearance $(P=.003)$ and clitoral function $(P=.02)$, and nonsignificantly lower ratings were reported for vaginal appearance $(P=.08)$ and function $(P=.32)$, respectively; see Table 1 . These findings were independent of whether the patient had been operated or not. However, as shown in Table 1, nonsignificantly lower ratings after surgical clitoral resections than clitoral reductions were observed. No significant correlations were found between age at surgery and rating of genitalia (data not shown), except a higher satisfaction with clitoral function the younger the age at surgery $\left(r_{s}=-0.45, P=.03\right)$. In patients, vaginal ratings correlated positively to vaginal diameter (appearance: $r_{s}=0.47[P=.03]$; function: $\left.r_{s}=0.42[P=.08]\right)$. Vaginal anatomy and function were rated by patients without transplants 7, that is, satisfied, while clitoral anatomy and 


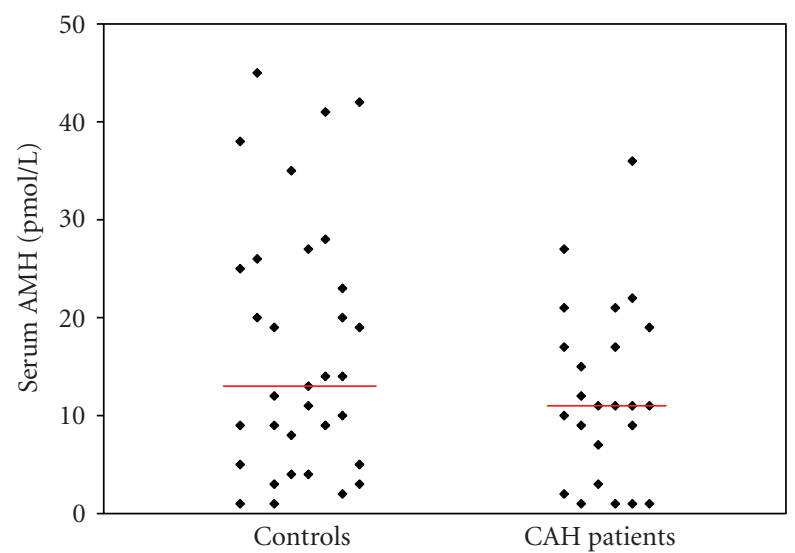

FIgURE 2: Serum levels of antimüllerian hormone (AMH) in patients with congenital adrenal hyperplasia $(\mathrm{CAH})$ and in controls. Lines indicate median values.

function in one patient was rated 6 and 2, respectively, and in the other as 7, respectively. Satisfaction with clitoral and vaginal appearance correlated negatively with the degree of virilisation at time of diagnosis $\left(r_{s}=-0.38[P=.05]\right.$ and $\left.r_{s}=-0.39[P=.04]\right)$.

The CAH patients had smaller uterine volume (median $29.1 \mathrm{ml}$ [range $7.5-56.7 \mathrm{ml}$ ]), as compared to the healthy controls $(47.4 \mathrm{ml}$ [15.9-177.5]), $P=.009$, Figure 1. In nullipara patients, uterine volumes (median $24.1 \mathrm{ml}$ [7.544.6], $n=11$ ) were smaller than in the control group (median $45.7 \mathrm{ml}$ [15.9-67.0], $n=14), P=.006$. There were no significant differences in uterine volumes in primipara/multipara patients (median $41.9 \mathrm{ml}$ [25.6-56.7], $n=5$ ) versus their controls (median 66.6 ml [25.6-177.5], $n=13$ ), $P=.59$. No associations were observed between uterine volume and androgen- or AMH-levels (data not shown).

A nonsignificant larger median ovarian volume was observed in the CAH group (4.4 $\mathrm{ml}$ [range $1.3-10.8 \mathrm{ml}$ ], $n=13)$, as compared to the controls (2.8 $\mathrm{ml}$ [0.6-10.8], $n=26), P=.09$; see Figure 1 . In the patients no associations were observed between ovarian volume and androgen- or AMH-levels (data not shown). There were no significant differences in levels of serum-AMH between patients (median $11.0 \mathrm{pmol} / \mathrm{L}$ [1-36]) and controls (13.0 pmol/L [1-45]), $P=.16$; see Figure 2. The AMH-levels in SW-, SV- and LO-patients of $11.0 \mathrm{pmol} / \mathrm{L}(1-36), 6.5 \mathrm{pmol} / \mathrm{L}(1-21)$, and $15 \mathrm{pmol} / \mathrm{L}$ (9-27), respectively, did not differ between $\mathrm{CAH}$ subgroups $(P=.58)$. The age at menarche was significantly later in patients (14.8 years, 9.0-19.8) than in controls (13.0 years $10.0-15.5), P=.001$.

$\mathrm{CAH}$ females had significantly higher hirsutism scores than matched controls $(2.0[0-12]$ versus 0.0 [0-11], $P=.001)$. In $\mathrm{CAH}$ subgroups hirsutism scores were $\mathrm{SW}=$ $2.0[0-12], \mathrm{SV}=2.0[1-4]$, and $\mathrm{LO}=2.0[0-9]$, reaching statistical significance in salt-wasters versus their controls $(P=.01)$. In CAH patients, positive correlations were observed between hirsutism and serum levels of testosterone $\left(r_{s}=.42, P=.03\right)$ and DHT $\left(r_{s}=.55, P=.003\right)$, respectively, while no significant correlation was observed to serum androstenedione $\left(r_{s}=.35, P=.08\right)$ and serum DHEAS $\left(r_{s}=.16, P=.45\right)$. Hirsutism did not correlate to serum AMH-level (data not shown). BMI did not differ between patients (25.0 [20.6-42.8]) and controls (26.7 [18.739.5]). A positive correlation was found between hirsutism score and BMI in patients alone $\left(r_{s}=0.36, P=.06\right)$ and when grouped together with the controls $\left(r_{s}=0.26\right.$, $P=.05)$.

As compared to the controls $(n=1)$, a significantly higher proportion of the patients $(n=13)$ had never had intercourse $(P=.001)$, and age at 1 st intercourse was higher $(18$ years [14-35] versus 16 years [13-19], $P=.02)$. In general, age at 1 st intercourse increased from nonclassical CAH (14 years [14-22] to classical CAH; i.e., SV: 18 years [16-20] and SW: 19 years [16-35]). Both parameters were positively associated with the degree of virilisation at birth $\left(r_{s}=.34, P=.06\right.$, and $r_{s}=0.52, P=.02$, resp.). Lack of intercourse was inversely correlated with vaginal diameter $\left(r_{s}=-0.70, P<.001\right)$, whether or not the patient had been operated $(P=.04)$, and positively with the number of vaginal operations $\left(r_{s}=0.37\right.$, $P=.03)$, while no associations were observed for vaginal or clitoral lengths or surgery of clitoris. Previous contact to a sexologist was reported in two CAH females and in none of the controls. However, a need for counselling was reported in seven CAH patients, and one control.

\section{Discussion}

The present study revealed significant differences in the size of internal and external genitalia between patients with congenital adrenal hyperplasia due to 21-hydroxylase deficiency and healthy women. The overall cosmetic results were satisfactory for the majority of patients. However, the lower rating of genital appearance and function in patients appeared to influence their sexual life.

In this study, the clitoral operations differed in type and number. The different surgery techniques may reflect historical trends, as clitoridectomy has been replaced by nerve-sparing clitoral reductions in the last decades [9]. Six CAH patients were operated twice. In one of these patients, the initial operation comprised a corporeal sparing clitoroplasty, while a reduction was performed in the second operation. However in the other patients, reoperation may have been performed because of regrowth of the clitoral corpora during exposure to high androgen levels either due to noncompliance or nonoptimal treatment. Current androgen levels were not correlated to any of the gynecological outcomes. This may be explained by the fact that current androgen status is highly dependent on current medical treatment and compliance of treatment of the $\mathrm{CAH}$ women whereas the herestudied outcome parameters are more influenced by prenatal or early postnatal androgen level and long-term treatment success. In some patients, androgen levels at the time of study entry were in fact suppressed to lower than normal values. In general, the patients had a vaginal depth within normal limits, but there was still a tendency to avoid or delay intercourse. Long-term surgical outcomes in patients with disorders of sexual development including $\mathrm{CAH}$ have reached discrepant conclusions $[10,11]$. 
It has been debated whether the optimal timing of surgery is in early infancy, during puberty, or as some patient support groups currently advocate when the patient can actively participate in the decision.

Our study demonstrated a significantly lower uterine volume in $\mathrm{CAH}$ women than in healthy controls, which was not completely ameliorated after successful pregnancy. The compromised uterine growth may reflect an imbalance between estrogens and androgens during adolescence, which additionally may contribute to the reported delayed time at menarche as seen in this study. Also a prenatal excess of androgens may affect uterine development permanently. In Turner syndrome, uterine hypoplasia is reported [12], and achievement of normal uterine growth during hormone replacement therapy in adolescence and early adulthood is likewise difficult. A suboptimal uterine development may contribute to the lower fertility rates seen in $\mathrm{CAH}$ females $[13,14]$, and may be associated with a higher prevalence of miscarriage and premature birth.

$\mathrm{CAH}$ patients had slightly larger ovarian volumes than their controls. This may reflect a higher prevalence of polycystic ovary syndrome, PCOS [15]; thus, both polycystic ovaries [16] and insulin resistance $[17,18]$ have been reported in CAH. However, in the present study, a suspicion of PCOS was not supported by elevated serum levels of antimüllerian hormone in the patients. In fact, AMH levels in our CAH patients tended to be lower than in age-matched controls and were associated with the degree of initial virilisation. Thus, it is tempting to speculate that prenatal androgen excess may reduce the number of preantral follicles in the ovary and thereby ovarian reserve in $\mathrm{CAH}$ patients. Clitoral length may be a marker of prenatal androgen levels, and thus, the observed inverse correlation between $\mathrm{AMH}$ levels and clitoral length additionally may indirectly reflect an inverse correlation between AMH and prenatal androgen excess. Prenatal androgen excess may contribute to the reduced fertility rates of $\mathrm{CAH}$ patients, which was also previously reported in our study group [3]. Hirsutism correlated to current serum levels of testosterone and DHT, but not to current AMH levels. Hirsutism mostly reflects hyperandrogenism, whereas AMH is more likely more related to the prenatal ovarian development and thereby the existing ovarian follicle pool than to current testosterone levels. Given the degree of hirsutism and delayed menarche, the patients most likely have been exposed to longer periods of hyperandrogenaemia, which may either be of adrenal or ovarian origin [19]. Ovarian hyperandrogenism in classic $\mathrm{CAH}$ is reported to result from perinatal masculinization of the hypothalamic-pituitary-ovarian axis [20]. With a BMI within the range of overweight [21], obesity in the $\mathrm{CAH}$ patients, and especially the higher risk of obesity in $\mathrm{CAH}$ children as compared to the background population [22], may further aggravate a hyperandrogenic condition. Another explanation for larger ovarian volumes in the CAH patients may be ovarian adrenal rest tumours, which may originate from pluripotent cells [23]. As the adrenals develop in the immediate vicinity of the gonads, some adrenal tissue may adhere to the gonads, and thereby descent with the testis or the ovary [24]. Adrenal rest tumours in female CAH patient have been inconsistently reported $[25,26]$, but are a wellknown phenomenon in male patients, in whom they may be associated with a risk of subfertility $[27,28]$. Unfortunately, our ultrasound equipment was not sensitive enough to allow detailed description of ovarian morphology.

We observed no significant correlations between current serum-antimüllerian hormone levels and vaginal measurements, uterine volume and ovarian volume, respectively. A possible explanation for the lacking correlation between these gynecological parameters and serum levels of AMH may include that the study group is small compared to large biological variation in vaginal, uterine and ovarian parameters. We have no obvious biological explanation for the observed inverse correlation between serum concentrations of AMH and age at clitoral surgery, which therefore needs to be supported by other studies.

Although around ninety percent of patients expressed satisfaction, especially clitoral appearance was rated significantly lower in patients than in controls, and more pronounced after clitoral resections than clitoral reductions. Furthermore, the initial degree of virilisation at birth was directly associated with older age at 1st intercourse and lower cosmetic rating. Dissatisfaction with genital outcome in 46,XY-females with micropenis reared as females [29], and sexual difficulties in DSD-patients including CAH [30], and in 46,XY-females after clitoral amputations compared to other types of clitoral operations [31], has been reported. Thus, it appears that the more conservative approach to clitoral surgery is generally better perceived by patients than previous clitoridectomies. A tendency for higher satisfaction with clitoral function was observed the younger the age at clitoral surgery. This may reflect that not only the type of surgery, but also the timing of surgery is important for development of the body image. This observation may thus add an important aspect to ongoing debates about the best timing of genital surgery from a medical and psychological view point. In the present study, the physician who collected the data was not the patients' usual consultant. Therefore we believe that there was no obvious compulsion to rate artificially high, and our results reflect the patients' true opinion.

As compared to healthy controls, a larger proportion of patients had never been engaged in intercourse, and age at 1st intercourse increased with increasing severity of the mutation. Similar findings have been reported, comparing $\mathrm{CAH}$ females to healthy controls $[14,32]$ and to nonaffected sisters [33], respectively. Only a small subgroup of patients had undergone sexological counseling, but a significant proportion of patients reported that they would like such counseling. It has been suggested that such counseling should start from preadolescence, when most girls need information about their condition to cope with hormonereplacement therapy and often vaginoplasty or dilatation [34]. Thus, in the present study, lack of intercourse was more frequent in women who were operated with a positive correlation to the total number of vaginal operations, and an inverse correlation to vaginal diameter. These observations strengthen the need of counseling, and it may also be advantageous that such counseling was available during 
adult life. To our knowledge no data exist about long-term outcome of vaginal operations over the entire life span of sexual activity.

Limitations of this study include that six patients $(\mathrm{SW}=4, \mathrm{SV}=2)$ declined gynecological examination, and gynecological examination was not performed in four SWpatients due to logistical reasons. We cannot exclude that especially the patients' refusal for examination may have caused a bias towards a better outcome. Furthermore, despite the matching with controls for age and school education, we cannot completely exclude selection bias: patients were recruited from a university hospital and may therefore not be representative for the entire $\mathrm{CAH}$ population. Additionally, the medical and surgical care of the included patients spans over several decades in which there have been significant changes in standard of care. Thus, our results may not be applicable to current treatments. The lower participation rate in the healthy controls may have introduced a bias towards inclusion of either controls with poorest outcomes (thereby giving the lowest estimate of difference between patients and controls) or controls with best outcomes (thereby overestimating the real difference between patients and controls). We have no data on nonparticipants among controls and are therefore unable to analyze this bias.

\section{Conclusion}

In the present study, long-term cosmetic and functional reproductive outcomes of women with congenital adrenal hyperplasia due to 21-hydroxylase deficiency were suboptimal. Prenatal hormonal and genetic factors may contribute to these outcomes, but better counseling of patients throughout adult life may improve long-term satisfaction. Given the complexity of CAH conditions, our findings support international recommendations that specialized multidisciplinary teams should be involved in the follow-up of these patients, not only during childhood and adolescence, but also during adult life.

\section{Acknowledgements}

This paper was supported by grants from the University of Copenhagen, the Danish Research Foundation (no. 22-010346), the Lundbeck Foundation (no. 44/01), the Dagmar Marshall Foundation, the Toyota Foundation (no. 880), the Glashoff's Foundation (no. 302499), and Mr. Knudsen's Award.

\section{References}

[1] P. C. Hindmarsh, "Management of the child with congenital adrenal hyperplasia," Best Practice and Research Clinical Endocrinology and Metabolism, vol. 23, no. 2, pp. 193-208, 2009.

[2] T. H. Johannsen, C. P. L. Ripa, J. M. Reinisch, M. Schwartz, E. L. Mortensen, and K. M. Main, "Impaired cognitive function in women with congenital adrenal hyperplasia," Journal of Clinical Endocrinology and Metabolism, vol. 91, no. 4, pp. 1376-1381, 2006.
[3] T. H. Johannsen, C. P. L. Ripa, E. L. Mortensen, and K. M. Main, "Quality of life in 70 women with disorders of sex development," European Journal of Endocrinology, vol. 155, no. 6, pp. 877-885, 2006.

[4] A. Wedell, A. Thilén, E. M. Ritzén, B. Stengler, and H. Luthman, "Mutational spectrum of the steroid 21-hydroxylase gene in Sweden: implications for genetic diagnosis and association with disease manifestation," Journal of Clinical Endocrinology and Metabolism, vol. 78, no. 5, pp. 1145-1152, 1994.

[5] S. Lenz, A. Giwercman, A. Elsborg et al., "Ultrasonic testicular texture and size in 444 men from the general population: correlation to semen quality," European Urology, vol. 24, no. 2, pp. 231-238, 1993.

[6] D. Ferriman and J. D. Gallwey, "Clinical assessment of body hair growth in women," The Journal of Clinical Endocrinology and Metabolism, vol. 21, pp. 1440-1447, 1961.

[7] R. Hatch, R. L. Rosenfield, M. H. Kim, and D. Tredway, "Hirsutism: implications, etiology, and management," American Journal of Obstetrics and Gynecology, vol. 140, no. 7, pp. 815830,1981 .

[8] G. Lykkesfeldt, P. Bennett, A. E. Lykkesfeldt, S. Micic, S. Møller, and B. Svenstrup, "Abnormal androgen and oestrogen metabolism in men with steroid sulphatase deficiency and recessive X-linked ichthyosis," Clinical Endocrinology, vol. 23, no. 4, pp. 385-393, 1985.

[9] J. M. Schober, "Long-term outcomes and changing attitudes to intersexuality," British Journal of Urology International, vol. 83, supplement 3, pp. 39-50, 1999.

[10] S. M. Creighton, C. L. Minto, and S. J. Steele, "Objective cosmetic and anatomical outcomes at adolescence of feminising surgery for ambiguous genitalia done in childhood," The Lancet, vol. 358, no. 9276, pp. 124-125, 2001.

[11] K. Newman, J. Randolph, and K. Anderson, "The surgical management of infants and children with ambiguous genitalia: lessons learned from 25 years," Annals of Surgery, vol. 215, no. 6, pp. 644-653, 1992.

[12] H. P. Haber and M. B. Ranke, "Pelvic ultrasonography in Turner syndrome: standards for uterine and ovarian volume," Journal of Ultrasound in Medicine, vol. 18, no. 4, pp. 271-276, 1999.

[13] N. M. M. L. Stikkelbroeck, A. R. M. M. Hermus, D. D. M. Braat, and B. J. Otten, "Fertility in women with congenital adrenal hyperplasia due to 21-hydroxylase deficiency," Obstetrical and Gynecological Survey, vol. 58, no. 4, pp. 275-284, 2003.

[14] F. Gastaud, C. Bouvattier, L. Duranteau et al., "Impaired sexual and reproductive outcomes in women with classical forms of congenital adrenal hyperplasia," Journal of Clinical Endocrinology and Metabolism, vol. 92, no. 4, pp. 1391-1396, 2007.

[15] S. Franks, "Polycystic ovary syndrome," The New England Journal of Medicine, vol. 333, no. 13, pp. 853-861, 1995.

[16] W. M. Hague, J. Adams, C. Rodda et al., "The prevalence of polycystic ovaries in patients with congenital adrenal hyperplasia and their close relatives," Clinical Endocrinology, vol. 33, no. 4, pp. 501-510, 1990.

[17] E. Charmandari, M. Weise, S. R. Bornstein et al., "Children with classic congenital adrenal hyperplasia have elevated serum leptin concentrations and insulin resistance: potential clinical implications," Journal of Clinical Endocrinology and Metabolism, vol. 87, no. 5, pp. 2114-2120, 2002.

[18] P. W. Speiser, J. Serrat, M. I. New, and J. M. Gertner, "Insulin insensitivity in adrenal hyperplasia due to nonclassical steroid 21-hydroxylase deficiency," Journal of Clinical Endocrinology and Metabolism, vol. 75, no. 6, pp. 1421-1424, 1992. 
[19] E. Carmina and R. A. Lobo, "Ovarian suppression reduces clinical and endocrine expression of late-onset congenital adrenal hyperplasia due to 21-hydroxylase deficiency," Fertility and Sterility, vol. 62, no. 4, pp. 738-743, 1994.

[20] R. B. Barnes, R. L. Rosenfield, D. A. Ehrmann et al., "Ovarian hyperandrogynism as a result of congenital adrenal virilizing disorders: evidence for perinatal masculinization of neuroendocrine function in women," Journal of Clinical Endocrinology and Metabolism, vol. 79, no. 5, pp. 1328-1333, 1994.

[21] National Institutes of Health, National Heart, Lung and Blood Institute, "Clinical guidelines on the identification, evaluation, and treatment of overweight and obesity in adults-the evidence report," Obesity Research, vol. 6, supplement 2, pp. 51S-209S, 1998.

[22] T. M. K. Völkl, D. Simm, C. Beier, and H. G. Dörr, "Obesity among children and adolescents with classic congenital adrenal hyperplasia due to 21-hydroxylase deficiency," Pediatrics, vol. 117, no. 1, pp. e98-e105, 2006.

[23] J. L. Rutgers, R. H. Young, and R. E. Scully, "The testicular 'tumor' of the adrenogenital syndrome. A report of six cases and review of the literature on testicular masses in patients with adrenocortical disorders," American Journal of Surgical Pathology, vol. 12, no. 7, pp. 503-513, 1988.

[24] E. V. Dahl and R. C. Bahn, "Aberrant adrenal cortical tissue near the testis in human infants," American Journal of Pathology, vol. 40, pp. 587-598, 1962.

[25] G. Russo, P. Paesano, G. Taccagni, A. Del Maschio, and G. Chiumello, "Ovarian adrenal-like tissue in congenital adrenal hyperplasia," The New England Journal of Medicine, vol. 339, no. 12, pp. 853-854, 1998.

[26] N. M. M. L. Stikkelbroeck, A. R. M. M. Hermus, D. Schouten et al., "Prevalence of ovarian adrenal rest tumours and polycystic ovaries in females with congenital adrenal hyperplasia: results of ultrasonography and MR imaging," European Radiology, vol. 14, no. 10, pp. 1802-1806, 2004.

[27] M. S. Cabrera, M. G. Vogiatzi, and M. I. New, "Long term outcome in adult males with classic congenital adrenal hyperplasia," Journal of Clinical Endocrinology and Metabolism, vol. 86, no. 7, pp. 3070-3078, 2001.

[28] N. M. M. L. Stikkelbroeck, B. J. Otten, A. Pasic et al., "High prevalence of testicular adrenal rest tumors, impaired spermatogenesis, and Leydig cell failure in adolescent and adult males with congenital adrenal hyperplasia," Journal of Clinical Endocrinology and Metabolism, vol. 86, no. 12, pp. 5721-5728, 2001.

[29] A. B. Wisniewski, C. J. Migeon, J. P. Gearhart et al., "Congenital micropenis: long-term medical, surgical and psychosexual follow-up of individuals raised male or female," Hormone Research, vol. 56, no. 1-2, pp. 3-11, 2001.

[30] C. L. Minto, L.-M. Liao, C. R. J. Woodhouse, P. G. Ransley, and S. M. Creighton, "The effect of clitoral surgery on sexual outcome in individuals who have intersex conditions with ambiguous genitalia: a cross-sectional study," The Lancet, vol. 361, no. 9365, pp. 1252-1257, 2003.

[31] C. J. Migeon, A. B. Wisniewski, J. P. Gearhart et al., "Ambiguous genitalia with perineoscrotal hypospadias in 46,XY individuals: long-term medical, surgical, and psychosexual outcome," Pediatrics, vol. 110, no. 3, p. e31, 2002.

[32] A. Nordenskjöld, G. Holmdahl, L. Frisén et al., "Type of mutation and surgical procedure affect long-term quality of life for women with congenital adrenal hyperplasia," Journal of Clinical Endocrinology and Metabolism, vol. 93, no. 2, pp. 380386, 2008.
[33] R. W. Dittmann, M. E. Kappes, and M. H. Kappes, "Sexual behavior in adolescent and adult females with congenital adrenal hyperplasia," Psychoneuroendocrinology, vol. 17, no. 23, pp. 153-170, 1992.

[34] F. M. E. Slijper, "Clitoral surgery and sexual outcome in intersex conditions," The Lancet, vol. 361, no. 9365, pp. 12361237, 2003. 